\title{
Monosomía 9p24 secundaria a translocación $(2 ; 9)$ en dos pacientes no relacionadas
} Monosomy $9 p 24$ in two non-related patients as result of a translocation $(2 ; 9)$

\author{
Dra. Nayla Y. León-Carlos ${ }^{a}$ Dra. Constanza García-Delgadoa, Biol. Ariadna B. Morales-Jiméneza, \\ Dr. Carlos Serrano-Bello ${ }^{b}$ M. en C. Alicia Cervantes ${ }^{c}$ y Dra. Verónica F. Morán Barroso ${ }^{a}$
}

\section{RESUMEN}

En pacientes con malformaciones congénitas y retraso del desarrollo psicomotor, deben descartarse cromosomopatías. Las más frecuentes son las translocaciones recíprocas balanceadas, presentes en 1:500 recién nacidos vivos. Por lo general, los portadores tienen fenotipo normal, aunque, ocasionalmente, presentan infertilidad, abortos o hijos con malformaciones. La translocación balanceada entre los cromosomas 2 y 9 puede originar descendencia con monosomías y trisomías de estos cromosomas. La monosomía del brazo corto del cromosoma 9 puede presentarse con trigonocefalia, dismorfias faciales, anomalías genitales y retraso del desarrollo psicomotor. En este trabajo, se revisaron las alteraciones de los cromosomas 2 y/o 9 en los cariotipos realizados en nuestra Institución en 2005-2014. Se presentan dos pacientes con monosomía 9p asociada a translocación $(2 ; 9)$. Las pacientes comparten datos de monosomía 9p24-pter; la correlación genotipo-fenotipo es compleja por el tamaño de los segmentos involucrados. Se resalta la importancia del diagnóstico cromosómico para el asesoramiento genético.

Palabras clave: monosomía 9p, translocación (2;9), trisomía 2q, anomalías congénitas, bandeo cromosómico.

\begin{abstract}
In patients with malformations and delayed psychomotor development it is important to discard chromosomopathies. Balanced reciprocal translocations are the most frequent chromosomopathies present in 1:500 livenewborns. In general, carriers have normal phenotype, but they may have infertility, abortions or children with congenital malformations. The reciprocal translocation between chromosomes 2 and 9 can lead to offspring with monosomies and trisomies of these chromosomes. Short arm monosomy of chromosome 9 may
\end{abstract}

a. Departamento de Genética, Hospital Infantil de México Federico Gómez.

b. Departamento de Patología, Hospital Infantil de México Federico Gómez.

c. Servicio de Genética, Hospital General de México Dr. Eduardo Liceaga/Facultad de Medicina, Universidad Nacional Autónoma de México, Ciudad de México, México.

\section{Correspondencia:}

Dra. Verónica F. Morán Barroso: vfmoran@himfg.edu.mx

Financiamiento: Ninguno.

Conflicto de intereses: Ninguno que declarar.

Recibido: 11-9-2017

Aceptado: 21-2-2018 present delayed psychomotor development, trigonocephaly, facial dysmorphia and genital abnormalities. We reviewed GTG karyotype records from our Institution to identify cases with chromosomes 2 and / or 9 alterations from 2005 to 2014. We describe two cases with monosomy $9 p$ secondary to a translocation between chromosomes 2 and 9. The patients share features of monosomy 9p24-pter, however the genotypephenotype correlation is complex due to the extension of the involved segments. We emphasize the importance of chromosomal diagnosis to offer genetic assessment.

Key words: monosomy 9p, translocation (2;9), trisomy 2q, congenital abnormalities, chromosome banding.

http: / / dx.doi.org/10.5546/aap.2018.e603

Cómo citar: León-Carlos NY, García-Delgado C, Morales-Jiménez $\mathrm{AB}$, et al. Monosomía 9p24 secundaria a translocación $(2 ; 9)$ en dos pacientes no relacionadas. Arch Argent Pediatr 2018;116(4):e603-e608.

\section{INTRODUCCIÓN}

En la consulta pediátrica, se evalúan pacientes con malformaciones congénitas y retraso en el desarrollo psicomotor (RDPM), alteraciones que pueden deberse a cromosomopatías. ${ }^{1}$ Las translocaciones recíprocas balanceadas, sin pérdida ni ganancia de material genético, son las aberraciones cromosómicas más frecuentes, presentes en 1:500 recién nacidos vivos. ${ }^{2}$ Por lo general, los portadores balanceados tienen fenotipo normal; sin embargo, pueden presentar infertilidad, abortos o descendencia con malformaciones congénitas por la formación de gametos desbalanceados. En ocasiones, los portadores tienen un fenotipo anormal, debido a un defecto monogénico por disrupción de un gen. ${ }^{3}$ La monosomía del brazo corto del cromosoma 9 (9p) es poco frecuente; se caracteriza por RDPM, trigonocefalia, dismorfias faciales y anomalías genitales. ${ }^{4,5}$ En el diagnóstico de las cromosomopatías, se utilizan diversos estudios, como el cariotipo con bandas $G$ por tripsina y tinción con giemsa (GTG) o la hibridación in situ con fluorescencia (fluorescent in situ hybridization; FISH, por sus siglas en inglés), que detecta aberraciones en regiones específicas. 
En este trabajo, se revisaron los resultados de cariotipos con bandas GTG de enero de 2005 a diciembre de 2014 para conocer la frecuencia de aberraciones estructurales de los cromosomas 2 y/o 9 en la población del Hospital Infantil de México Federico Gómez (HIMFG). Se reportaron 5762 cariotipos, con 22 resultados que involucraban los cromosomas 2 y/o $9(0,38 \%)$. De ellos, tres tuvieron alteraciones del cromosoma 2 (una duplicación parcial del brazo largo -2q31-q33- y dos derivados de translocaciones); 16 mostraron alteración del cromosoma 9 y correspondieron a 8 derivados de una translocación, 5 deleciones, 3 de ellas de 9p; un anillo, una duplicación de brazo largo (9q) y uno con material adicional. Se identificó un caso que implicaba el cromosoma 2 y una inversión pericéntrica del cromosoma 9, y se expusieron dos casos con monosomía $9 \mathrm{p}$ secundaria a una translocación entre los cromosomas 2 y 9; se realizó un estudio clínico interdisciplinario y citogenético molecular.

\section{DESCRIPCIÓN DE CASOS CLÍNICOS Caso 1}

Recién nacida de padres jóvenes, sanos, no consanguíneos (Figura 1.A). Embarazo con esquema de maduración pulmonar con corticoesteroides por amenaza de parto pretérmino. Obtenida por cesárea a las 38 semanas de gestación con peso de $2550 \mathrm{~g}$ (percentil 3), talla de $45 \mathrm{~cm}$ (percentil 3) y puntaje de Apgar 6/8. Fue referida a los 6 días de vida por una cardiopatía congénita. Al momento de la exploración física,
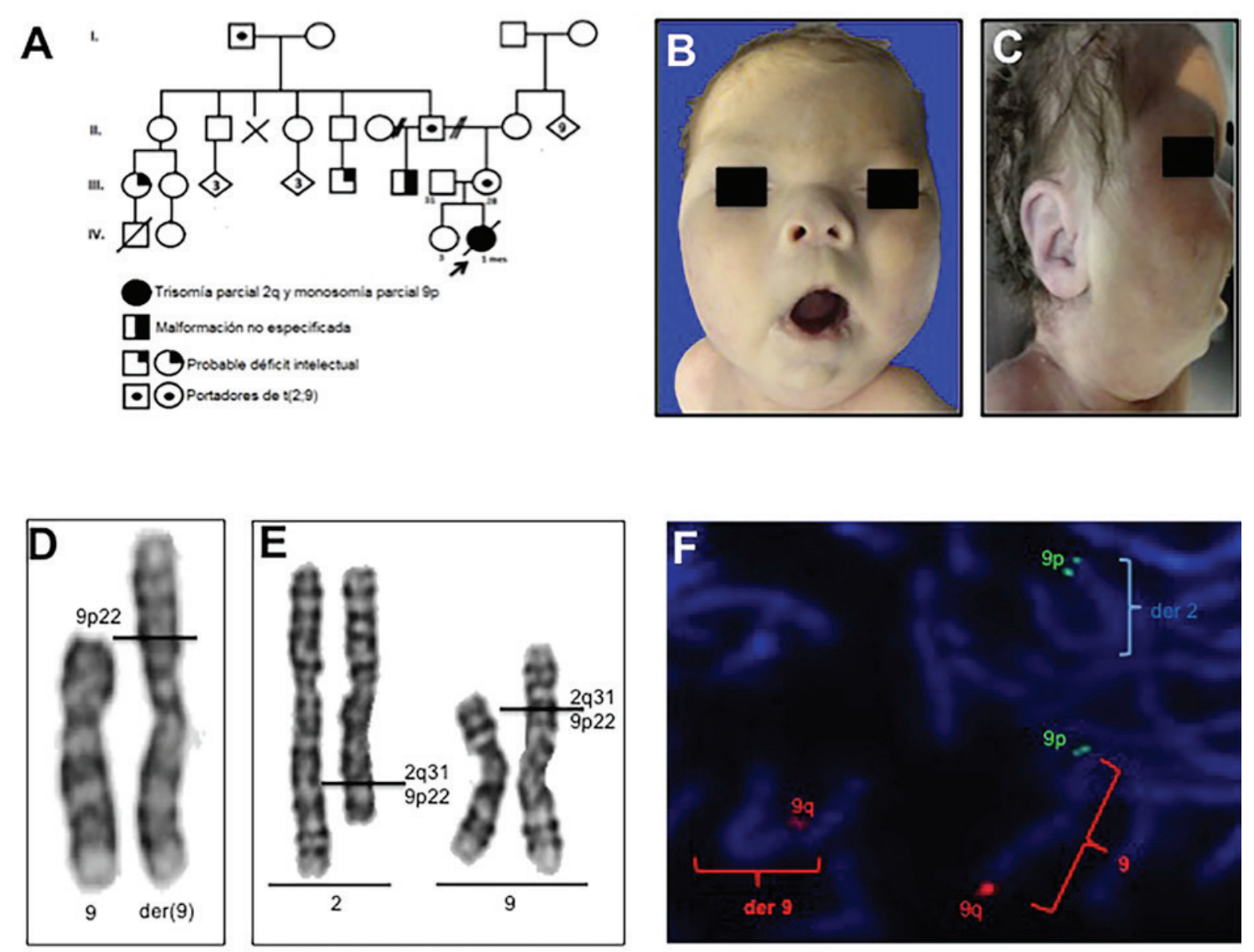

A: Árbol genealógico de la propósita. B, C: Fenotipo, nótese frontal prominente y puente nasal deprimido. Cariotipos parciales con bandas GTG: D, de la paciente, que muestra material adicional en el cromosoma 9, y E, de la madre, que muestra la translocación balanceada entre los cromosomas 2 y 9 . F. Análisis por FISH de una metafase parcial del abuelo materno. Se indican los cromosomas derivativos y el cromosoma 9 normal con las sondas subteloméricas de 9p (verde) y de 9q (roja) (ToTelVysion ${ }^{\mathrm{TM}}$, Vysis Abbott Laboratories, Abbott Park, ILL, USA). 
presentó braquicefalia, región frontal prominente, microftalmia y enoftalmos bilaterales, puente nasal deprimido, narinas antevertidas y cortas, paladar hendido central, pabellones auriculares dismórficos con hélix hipoplásico, soplo holosistólico y genitales externos femeninos (Figuras 1.B y 1.C). Se diagnosticó catarata congénita bilateral y coartación de aorta. Falleció a los 36 días de edad por complicaciones cardíacas.
El estudio post mórtem demostró agenesia del cuerpo calloso, comunicación interventricular y doble arteria renal bilateral (Tabla 1). El cariotipo mostró material adicional de origen desconocido en $9 \mathrm{p}$, que fue 46,XX, add(9)(p22) (Figura 1.D). El cariotipo del padre fue normal y el de la madre fue $46, X X, t(2 ; 9)(q 31 ; p 22)$, por lo que era portadora de una translocación balanceada entre los cromosomas 2 y 9 (Figura 1.E). El cariotipo

TABla 1. Características clínicas de la monosomía 9p24.3 reportadas y comparación con nuestras pacientes

\begin{tabular}{|c|c|c|c|c|c|c|}
\hline Características clínicas & Veitia et al. ${ }^{12}$ & Vinci et al. ${ }^{13}$ & Hauge et al. ${ }^{11}$ & Onesimo et al. ${ }^{8}$ & Caso 1 & Caso 2 \\
\hline Segmento de monosomía & 9p24.2-pter & 9p24.1-pter & 9pter & 9p24.1-pter & 9p22-pter & 9p24-pter \\
\hline Extensión de la deleción & NR & $6.7-7.1 \mathrm{Mb}$ & $1 \mathrm{Mb}$ & 6.5 & & \\
\hline Sexo cromosómico & $\mathrm{XY}$ & $X Y$ & $X Y$ & $X Y$ & $X X$ & $X X$ \\
\hline Género & NR & Masculino & Masculino & $\begin{array}{l}\text { Reasignación } \\
\text { Masculino }\end{array}$ & Femenino & Femenino \\
\hline Edad & 10 años & 11 años & NR & 8 meses & 36 días & $\begin{array}{l}5 \text { años y } \\
11 \text { meses }\end{array}$ \\
\hline Origen & Paterno & De novo & NR & NR & Materno & De novo \\
\hline $\begin{array}{l}\text { Técnica de análisis } \\
\text { cromosómico }\end{array}$ & Cariotipo & aCGH & FISH/aCGH & aCGH/FISH & $\begin{array}{l}\text { Cariotipo } \\
\text { con bandas } \\
\text { GTG }\end{array}$ & $\begin{array}{l}\text { Cariotipo } \\
\text { con bandas } \\
\text { GTG/FISH }\end{array}$ \\
\hline Plagiocefalia & NR & NR & NR & NR & - & + \\
\hline Frente prominente & - & - & + & + & + & - \\
\hline Depresión bitemporal & - & - & - & + & + & - \\
\hline Hipoplasia mediofacial & - & - & + & + & - & - \\
\hline Cejas arqueadas & - & - & + & + & - & + \\
\hline Fisuras palpebrales cortas/epicanto & to & - & + & + & - & - \\
\hline Fisuras palpebrales ascendentes & NR & NR & NR & NR & - & + \\
\hline Estrabismo & - & - & - & - & - & + \\
\hline Narinas antevertidas & - & - & + & - & + & + \\
\hline Punta nasal bulbosa & - & - & + & + & - & + \\
\hline Anomalía de pabellones auriculare & res - & - & + & + & + & + \\
\hline Filtrum largo & - & - & + & + & + & + \\
\hline Labio superior delgado & - & - & + & + & + & + \\
\hline Microrretrognatia & - & - & + & + & + & - \\
\hline Cuello corto & - & - & NR & + & - & - \\
\hline Teletelia & NR & NR & NR & NR & - & + \\
\hline Clinobraquidactilia & - & - & + & - & - & - \\
\hline Dedos largos & NR & NR & NR & NR & - & + \\
\hline Trastorno de conducta & + & + & + & + & NV & - \\
\hline Malformación del SNC & NR & NR & NR & NR & + & + \\
\hline RDPM & - & + & + & + & NV & + \\
\hline Hipotonía & - & - & + & - & - & - \\
\hline Convulsiones & - & - & - & + & - & - \\
\hline Anomalía cardíaca & - & - & - & + & + & - \\
\hline Genitales externos ambiguos & + & + & NR & + & - & - \\
\hline Disgenesia gonadal & + & + & NR & + & - & - \\
\hline Otros datos & & & & & Catarata, $\mathrm{PH}$ & Talla baja \\
\hline
\end{tabular}

NR: no reportado; NV: no valorable; PH: paladar hendido; aCGH: hibridación genómica comparativa por microarreglos; FISH: hibridación in situ con fluorescencia; GTG: bandas G por tripsina y tinción con giemsa; SNC: sistema nervioso central; RDPM: retraso en el desarrollo psicomotor. 
final de la paciente fue 46,XX, $\operatorname{der}(9) \mathrm{t}(2 ; 9)(\mathrm{q} 31 ; \mathrm{p} 22)$ mat. El abuelo y el bisabuelo maternos resultaron portadores de la translocación y, en ellos, se realizó un análisis con FISH (ToTelVysion, Vysis, Abbott Park, ILL, USA) (Figura 1.F).

\section{Caso 2}

Niña de 5 años y 11 meses de edad, tercera gestación de padres jóvenes, sanos, no consanguíneos; hermana de 11 años de edad con parálisis cerebral infantil. Embarazo con amenaza de aborto a los tres meses, infección de vías urinarias en el quinto mes y circular de cordón al cuello diagnosticada por ecografía obstétrica. Obtenida por cesárea a las 35 semanas de gestación con un peso de $2780 \mathrm{~g}$ (percentil 10), talla de $46 \mathrm{~cm}$ (percentil 10) y puntaje de Apgar 8/9. Referida por retraso de lenguaje afásico y moderado del desarrollo, atrofia cortical diagnosticada por resonancia magnética de encéfalo, talla baja y endotropia bilateral. Al momento de la exploración física, se encontró peso de $18,5 \mathrm{~kg}$ (percentil 10), talla de $107 \mathrm{~cm}$ (percentil 3), edad aparente menor que la cronológica, cráneo tendiente a la plagiocefalia, cejas pobladas y arqueadas, fisuras palpebrales ascendentes, punta nasal bulbosa, narinas antevertidas, hoyuelos retroauriculares izquierdos, filtrum largo, labios delgados, teletelia y genitales externos femeninos (Figuras 2.A y 2.B) (Tabla 1). El cariotipo reportó una translocación aparentemente balanceada entre los brazos cortos de los cromosomas 2 y 9: 46, XX,t $(2 ; 9)$ (p10;p24) (Figura 2.C). El cariotipo de ambos padres fue normal, por lo que la alteración se consideró de novo. El análisis por FISH con sondas subteloméricas de los cromosomas 2 y 9 en metafases de la propósita demostró la ausencia de la región subtelomérica $9 \mathrm{p}$ con cariotipo 46,XX,t(2;9)(p10;p24)del(9)(p24) (Figura 2.D), que correspondió a un rearreglo no balanceado: la región q31-qter del brazo largo del cromosoma 2

Figura 2. Caso 2.
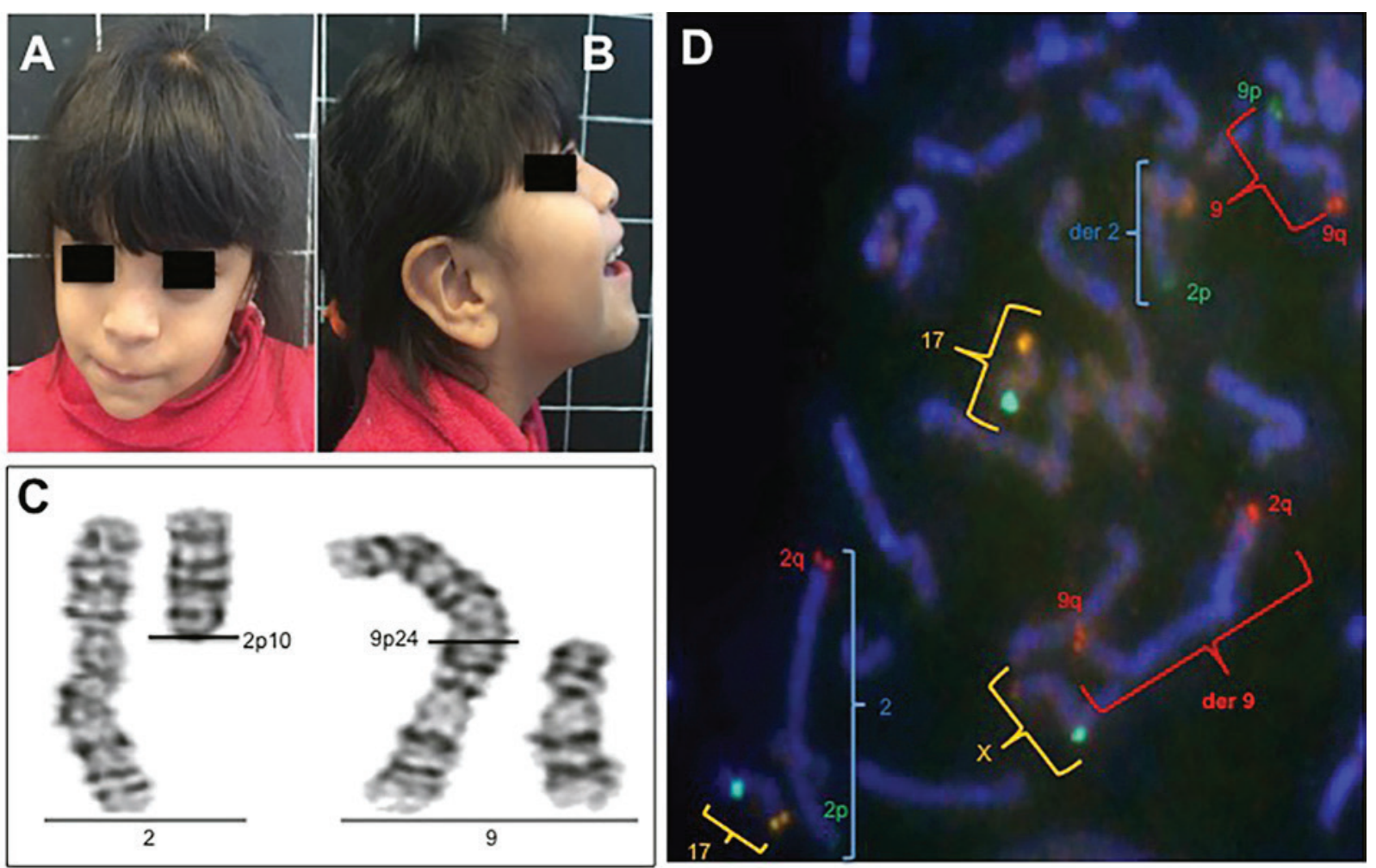

A, B: Fenotipo de la propósita; nótense las fisuras palpebrales ascendentes, punta nasal bulbosa, narinas antevertidas, filtrum largo y labios delgados. C: Cariotipo parcial con técnica de bandas GTG de la propósita. Se muestran la translocación t(2;9) y los puntos de ruptura. D: Análisis por FISH con sondas subteloméricas de los cromosomas 2 y 9 (ToTelVysion ${ }^{\text {TM }}$, Vysis Abbott Laboratories, Abbott Park, ILL, USA). Se señalan los cromosomas 2 y 9 normales y los derivativos que corresponden a la $t(2 ; 9)$ con señales verdes para 2 p y 9 p y, en rojo, para $2 q$ y 9q, que demuestran la pérdida de la región 9p24-pter. 
se translocó a la región distal del brazo corto del cromosoma 9 con deleción de la región p24-pter de este último.

\section{DISCUSIÓN}

La frecuencia de alteraciones de los cromosomas 2 y 9 en el período analizado fue baja, con más alteraciones del 9 en una relación 4:1. La monosomía 9p presenta RDPM, trigonocefalia, puente nasal deprimido, filtrum largo, cuello corto y alado, y anomalías genitales. Las alteraciones genitales y / o de gónadas se han observado en individuos masculinos con la deleción de 9p24.3-pter, que incluye los genes DMRT. ${ }^{6}$ Establecer la correlación genotipo-fenotipo para la monosomía 9p es compleja por la variación en los puntos de ruptura y la participación de regiones trisómicas derivadas de otros cromosomas. ${ }^{7,8} \mathrm{La}$ paciente 1 presentó malformaciones congénitas por ganancia de material genético en 2q31-2qter y pérdida en 9p22-9pter, alteraciones secundarias a la formación de un ovocito desbalanceado por segregación adyacente 1 de la translocación materna. La trisomía $2 q$ presenta anormalidades craneofaciales (braquicefalia, región frontal prominente, hipertelorismo, puente nasal deprimido, narinas antevertidas, filtrum largo, pabellones auriculares de implantación baja, comisuras labiales descendentes, micrognatia) y alteraciones sistémicas, como RDPM, hipotonía, malformaciones cardíacas, urinarias y del tracto gastrointestinal. ${ }^{9}$ El fenotipo de la paciente correspondió a la trisomía 2q, con braquicefalia, región frontal prominente, comisuras labiales descendentes y alteraciones cardíacas y urinarias, que resultó letal por la extensión del material trisómico del cromosoma 2. La paciente también presentó fenotipo compatible con la monosomía 9 p; sin embargo, no tuvo trigonocefalia, hipertelorismo, epicanto, cuello corto y alado, hipotonía ni alteraciones genitales.

En el segundo caso, la paciente tuvo una monosomía 9p24 pura. Las características en la paciente y en los casos ya reportados ${ }^{7,10-12}$ fueron RDPM, cejas arqueadas, narinas antevertidas, punta nasal bulbosa, anomalía de pabellones auriculares, filtrum largo y labio superior delgado. La paciente también presentó talla baja, plagiocefalia, fisuras palpebrales ascendentes, estrabismo, teletelia, dedos largos y malformación del sistema nervioso central.

En los dos casos aquí descritos, hubo pérdida de 9p24, región relacionada con alteraciones del desarrollo sexual por genes sensibles a dosis, como DMRT, necesarios para la formación de testículos en individuos $X Y$ y para la función ovárica en individuos XX. ${ }^{12}$ DMTR tiene expresión gonadal sexual específica; el desarrollo testicular alterado y la feminización XY ocurren cuando este gen se encuentra en estado hemicigoto. ${ }^{13}$ En las pacientes expuestas, no hubo alteraciones genitales y/o gonadales, lo que podría deberse a que estas son más frecuentes en individuos masculinos. ${ }^{6,14}$

No se ha encontrado alguna característica clínica presente en todos los casos de monosomía 9p24. ${ }^{710-12}$ Las pacientes expuestas compartían la malformación del sistema nervioso central, narinas antevertidas, anomalías en pabellones auriculares y filtrum largo. Su comparación es complicada, ya que, en el primer caso, la región perdida de 9 p era más extensa y su fenotipo se debía en forma predominante a la trisomía 2q31-qter. Es interesante que, en esta revisión, se hayan identificado dos casos de monosomía $9 p$ secundarias a una translocación con el cromosoma 2, pues son poco frecuentes. . $^{6,710-12,15}$

El asesoramiento genético del caso 1 con madre portadora balanceada de t $(2 ; 9)$ incluyó el riesgo teórico por embarazo de presentar abortos (33\%), hijos con malformaciones por monosomías o trisomías relacionadas con la translocación $(33 \%)$, portadores balanceados de la translocación sanos en fenotipo (16\%) o sanos en genotipo y fenotipo (16\%). El riesgo de recurrencia para el caso 2 fue similar al de la población general por ser de novo.

\section{CONCLUSIONES}

Las alteraciones estructurales de los cromosomas 2 y/o 9 en nuestra Institución fueron pocas, con mayor frecuencia en el cromosoma 9 y derivados de translocaciones recíprocas. El análisis de nuestros casos amplía la correlación genotipo-fenotipo de la monosomía 9 p con nuevos datos clínicos, como talla baja, plagiocefalia, fisuras palpebrales ascendentes, estrabismo, teletelia, dedos largos y malformación del sistema nervioso central. Se resalta la importancia del diagnóstico cromosómico para el manejo y asesoramiento genético de estos casos.

\section{REFERENCIAS}

1. Martin CL, Ledbetter D. Chromosomal Microarray Testing for Children with Unexplained Neurodevelopmental Disorders. JAMA 2017; 317(24):2545.

2. Kaiser-Rogers K, Rao KW. Structural chromosome rearrangements. En: GersenS, KeagleM(eds.). The Principles of Clinical Cytogenetics. 3th ed. New York: Springer; 2013. Págs.159-61. 
3. Midro AT, Zollino M, Wiland E, et al. Meiotic and pedigree segregation analyses in carriers of $\mathrm{t}(4 ; 8)(\mathrm{p} 16 ; \mathrm{p} 23.1)$ differing in localization of breakpoint positions at $4 \mathrm{p}$ subband $4 \mathrm{p} 16.3$ and 4p16.1. J Assist Reprod Genet 2016; 33(2):189-97.

4. Swinkels ME, Simons A, Smeets DF, et al. Clinical and cytogenetic characterization of 13 Dutch patients with deletion $9 p$ syndrome: Delineation of the critical region for a consensus phenotype. Am J Med Genet A 2008; 146A(11):1430-8.

5. Durmaz CD, Yararbas K, Kutlay NY, et al. Unusual Chromosomal Rearrangement Resulted in Interstitial Monosomy 9p: Case Report. Cytogenet Genome Res 2016; 148(1):19-24.

6. Barbaro M, Balsamo A, Anderlid BM, et al. Characterization of deletions at $9 \mathrm{p}$ affecting candidate regions for sex reversal and deletion 9p syndrome by MLPA. Eur J Med Genet 2009; 17(11):1439-47.

7. Onesimo R, Orteschi D, Scalzone M, et al. Chromosome $9 p$ deletion syndrome and sex reversal: Novel findings and redefinition of the critically deleted regions. Am J Med Genet A 2012; 158A(9):2266-71.

8. Sirisena ND, Wijetunge UK, de Silva $R$, et al. Child with deletion $9 p$ syndrome presenting with craniofacial dysmorphism, developmental delay, and multiple congenital malformations. Case Rep Genet 2013; 2013:785830.
9. Angle B, Hersh JH, Yen F, et al. Case of partial duplication $2 q 3$ with characteristic phenotype: rare occurrence of an unbalanced offspring resulting from a parental pericentric inversion. Am J Med Genet 2000; 91(2):126-30.

10. Hauge X, Raca G, Cooper S, et al. Detailed characterization and clinical correlations in 10 patients with distal deletions of chromosome 9p. Genet Med 2008; 10(8):599-611.

11. Veitia RA, Nunes M, Quintana-Murci L, et al. Swyer syndrome and $46, \mathrm{XY}$ partial gonadal dysgenesis associated with 9p deletions in the absence of monosomy-9p syndrome. Am J Hum Genet 1998; 63(3):901-5.

12. Vinci G, Chantot-BastaraudS, ElHouate B, etal. Association of deletion 9p, 46, XY gonadal dysgenesis and autistic spectrum disorder. Mol Hum Reprod 2007; 13(9):685-9.

13. Smith CA, McClive PJ, Western PS, et al. Conservation of a sex-determining gene. Nature 1999; 402(6762):601-2.

14. Abreu LS, Brassesco MS, Moreira ML, et al. Case report. Familial balanced translocation leading to an offspring with phenotypic manifestations of 9p syndrome. Genet Mol Res 2014; 13(2):4302-10.

15. Ledig S, Hiort O, Scherer G, et al. Array-CGH analysis in patients with syndromic and non-syndromic $X Y$ gonadal dysgenesis: Evaluation of array CGH as diagnostic tool and search for new candidate loci. Hum Reprod 2010; 25(10): 2637-46. 